\section{Role of Aqueductal CSF Stroke Volume in Idiopathic Normal-Pressure Hydrocephalus}

I read with great interest the article by Scollato et al entitled "Changes in Aqueductal CSF Stroke Volume in Shunted Patients with Idiopathic Normal-Pressure Hydrocephalus." In this study, the authors investigated whether there is a relationship between clinical outcome and changes in aqueductal CSF stroke volume (ACSV) in patients with idiopathic normal-pressure hydrocephalus (INPH) who have undergone shunting. I would like to thank the authors for presenting such a comprehensive study performed in a large series.

INPH is generally seen in the elderly and is characterized by gait disturbance, dementia, and urinary incontinence with normal opening pressure at lumbar puncture in patients without causative disorders and ventricular enlargement due to disturbed CSF circulation. ${ }^{2}$ Some symptoms may resolve with CSF diversion, so there are studies aimed at identifying patients who would respond to shunt treatment. ${ }^{2-5}$

Scollato et $\mathrm{al}^{1}$ showed that "INPH shunt responders presented higher preshunt ACSV values and shorter onset times of symptoms with respect to nonresponders." They also described an increase of ACSV (reversible phase) in the early period and a decrease in the late period among patients with INPH in their previous study. ${ }^{6}$ In this study, low ACSV values in unshunted patients were noted to be an indicator of irreversible neuronal damage occurring secondary to ischemia. ${ }^{6}$ The underlying cause of cerebral ischemia has been noted to be the reduction of flow in periventricular arterioles due to tangential and radial shearing force developing secondary to progressive ventriculomegaly. ${ }^{1,6,7}$ After the shunt therapy, the pressure reflected on periventricular arterioles decreases and cerebral ischemia resolves. ${ }^{8-10}$ Increase in cerebral perfusion occurring after the shunt may explain the clinical improvement in these patients. ${ }^{1}$

Although in many studies cerebral ischemia is centered in the pathophysiology of INPH, ischemia is not present in all INPH cases. ${ }^{8,9,11-13}$ Owler et $\mathrm{al}^{14}$ in their study with MR imaging and positronemission tomography reported that cerebral blood flow (CBF) is $19 \%$ decreased in patients with INPH compared with the control group. However in this study, the SD of the data was high, and CBF was normal in $16 \%$ of patients with INPH. The CBF measurements in patients with INPH and the control group suggest that ischemia is not a prerequisite for generation of INPH. ${ }^{9,15}$ It is not known if ischemia is the cause or the effect of the disease. ${ }^{13,16}$ The general concept is that decreased CBF causes neuronal loss. ${ }^{8}$ In the literature, it is reported that CBF is normal in $15 \%$ of patients with INPH. ${ }^{15,17}$ In patients with low CBF (global ischemia), a shunt procedure does not always increase $\mathrm{CBF}$ and no significant correlation has been shown between relief of symptoms and CBF. ${ }^{15}$

ACSV measurement is a noninvasive test that benefits INPH diagnosis. ${ }^{3,4,18,19}$ The recent article of Scollato et al ${ }^{1}$ showed that "INPH shunt responders presented higher preshunt ACSV values and shorter onset times of symptoms with respect to nonresponders." Also, Kim et $\mathrm{al}^{19}$ determined a decrease in CSF stroke volume among patients with INPH following shunt therapy. In their recent report, Scollato et al stated, "This study shows that ACSV may be a useful noninvasive tool for the preoperative selection of patients with INPH." Unfortunately, the relationship between the response to shunt treatment and ACSV value could not be fully understood. ${ }^{20}$ ACSV and velocity values have been reported to be inefficient in the prediction of shunt response by several authors. ${ }^{3,4,18}$ While the drop in ACSV values appears to be higher in patients benefiting from shunt than in those who do not, no definitive ACSV limit value has been described for deciding to perform a shunt treatment. ${ }^{1,3,4,9,18,19}$

In daily practice, the decision to perform a shunt treatment is based on clinical/laboratory findings and lumbar drainage results. However, do Scollato et $\mathrm{al}^{1}$ recommend proceeding with serial ACSV measurements to determine whether to apply shunt treatment in such cases? In patients who show increasing ACSV values, applying a CSF diversion may be a wise step in terms of preventing ischemia and atrophy while the brain is in a reversible phase, but how should we treat patients who demonstrate gradually decreasing ACSV values in the serial ACSV measurements? Considering that these patients are already in the irreversible phase, should we totally dismiss the idea of shunt treatment or should we apply it and give those patients a chance? Moreover, in the postoperative period, one should bear in mind that time will be lost performing serial ACSV measurements; thus the unshunted patients will advance closer to the irreversible phase. Keeping the above-mentioned information in mind, I believe that baseline ACSV and CSF velocity values should be obtained in the preoperative period in all patients with INPH; however, in my opinion, those baseline values are not useful in predicting shunt response. Similarly, although we can say that patients who have just begun to show symptoms have a better response to shunt treatment, the role of this knowledge on deciding shunt treatment is limited in clinical practice.

Nonetheless, I agree with the conclusion of Scollato et $\mathrm{al}^{1}$ that ACSV measurements are useful in monitoring the response following shunt treatment. Moreover, as mentioned by Scollato et al, I believe that ACSV measurements can be beneficial in the evaluation of shunt function and in the detection of the complications such as subdural fluid collection.

In light of the results of the study conducted of Scollato et al, ${ }^{1} \mathrm{I}$ think new studies should focus on the correlation between ACSV and cerebral perfusion. Those studies may shed light on whether ischemia is the cause or outcome of INPH.

In conclusion, despite hundreds of studies conducted in the past years, investigators have failed to reveal the etiopathogenesis and shunt indication of INPH disease. Currently, there is no test that can establish a definitive diagnosis or predict shunt response. Determination of ACSV is noted as a method that is helpful in INPH diagnosis and therapy monitoring. I believe that to determine phase-contrast MR imaging parameters that can predict the shunt response, further more comprehensive studies with larger series are needed.

\section{References}

1. Scollato A, Gallina P, Gautam B, et al. Changes in aqueductal CSF stroke volume in shunted patients with idiopathic normal-pressure hydrocephalus. AJNR Am J Neuroradiol 2009;30:1580-86. Epub 2009 May 20

2. Algın O, Hakyemez B, Taskapilioglu O, et al. Morphologic features and flow void phenomenon in normal pressure hydrocephalus and other dementias: are they really significant? Acad Radiol 2009;16:1373-80

3. Kahlon B, Annertz M, Ståhlberg F, et al. Is aqueductal stroke volume, measured with cine phase-contrast magnetic resonance imaging scans useful in predicting outcome of shunt surgery in suspected normal pressure hydrocephalus? Neurosurgery 2007;60:124-30

4. Dixon GR, Friedman JA, Luetmer PH, et al. Use of cerebrospinal fluid flow rates measured by phase-contrast MR to predict outcome of ventriculo-peritoneal shunting for idiopathic normal-pressure hydrocephalus. Mayo Clin Proc 2002;77:509-14

5. Bradley WG. Cerebrospinal fluid dynamics and shunt responsiveness in patients with normal-pressure hydrocephalus. Mayo Clin Proc 2002;77:507-08

6. Scollato A, Tenenbaum R, Bahl G, et al. Changes in aqueductal CSF stroke volume and progression of symptoms in patients with unshunted idiopathic normal pressure hydrocephalus. AJNR Am J Neuroradiol 2008;29:193-98

7. Mathew NT, Meyer JS, Hartmann A, et al. Abnormal cerebrospinal fluid-blood 
flow dynamics: implications in diagnosis, treatment, and prognosis in normal pressure hydrocephalus. Arch Neurol 1975;32:657-64

8. Brooks DJ, Beaney RP, Powell M, et al. Studies on cerebral oxygen metabolism, blood flow and blood volume, in patients with hydrocephalus before and after surgical decompression, using positron emission tomography. Brain 1986; 109:613-28

9. Bradley WG. Idiopathic normal pressure hydrocephalus: new findings and thoughts on etiology. AJNR Am J Neuroradiol 2008;29:1-3

10. De Marco G, Peretti II, Poncelet AD, et al. Intracranial fluid dynamics in normal and hydrocephalic states: systems analysis with phase-contrast magnetic resonance imaging. J Comput Assist Tomogr 2004;28:247-54

11. Edwards RJ, Dombrowski SM, Luciano MG, et al. Chronic hydrocephalus in adults. Brain Pathol 2004;14:325-36

12. Momjain S, Owler BK, Czosnyka Z, et al. Pattern of white matter regional cerebral blood flow and autoregulation in normal pressure hydrocephalus. Brain 2004;127:965-72

13. Bradley WG. Normal pressure hydrocephalus and deep white matter ischemia: which is the chicken, and which is the egg? AJNR Am J Neuroradiol 2001;22:1638-40

14. Owler BK, Momjain S, Czosnyka Z, et al. Normal pressure hydrocephalus and cerebral blood flow: a PET study of baseline values. J Cereb Blood Flow Metab 2004;24:17-23

15. Bateman GA. The pathophysiology of idiopathic normal pressure hydro- cephalus: cerebral ischemia or altered venous hemodynamics? AJNR Am J Neuroradiol 2008;29:198-203

16. Krauss JK, Havle B. Normal pressure hydrocephalus: survey of contemporary diagnostic algorithms and therapeutic decision-making in clinical practice. Acta Neurochir (Wien) 2004;146:379-88. Epub 2004 Feb 27

17. Fukuhara T, Luciano MG. Clinical features of late-onset idiopathic aqueductal stenosis. Surg Neurol 2001;55:132-37

18. Bateman GA, Levi CR, Schofield P, et al. The pathophysiology of the aqueduct stroke volume in normal pressure hydrocephalus: can co-morbidity with other forms of dementia be excluded? Neuroradiology 2005;47:741-48

19. Kim DS, Choi JU, Huh R, et al. Quantitative assessment of cerebrospinal fluid hydrodynamics using a phase contrast cine MR image in hydrocephalus. Childs Nerv Syst 1999;15:461-67

20. El Sankari SS, Baledent O, Gondry-Jouet C, et al. Aging effects on cerebra blood and cerebrospinal fluid flows. J Cereb Blood Flow Metab 2007;27:156372. Epub 2007 Feb 21

O. Algin

Department of Radiology Uludag University Medical Faculty

Gorukle, Bursa, Turkey

DOI 10.3174/ajnr.A1943 